Rapid Reviews COVID-19

\title{
Review 1: "LOW BIRTH WEIGHT AS A RISK FACTOR FOR SEVERE COVID-19 IN ADULTS"
}

Stephanie Eick ${ }^{1}$, Naida Gaber ${ }^{2}$

${ }^{1}$ University of California San Francisco, Obstetrics, Gynecology and Reproductive Sciences, 2University of California, San Francisco

Published on: Dec 03, 2020

DOI: $10.1162 / 2 \mathrm{e} 3983 f 5 . f 2948 \mathrm{f} 20$

License: Creative Commons Attribution 4.0 International License (CC-BY 4.0). 


\section{RR:C19 Evidence Scale rating by reviewer:}

Not informative. The flaws in the data and methods in this study are sufficiently serious that they do not substantially justify the claims made. It is not possible to say whether the results and conclusions would match that of the hypothetical ideal study. The study should not be considered as evidence by decision-makers.

$* * * * * * * * * * * * * * * * * * * * * * * * * * * * * * * * * * * * * * *$

\section{Review:}

The manuscript entitled "Low birth weight as a risk factor for severe COVID-19 in adults" was published on medRxiv on September 15, 2020. In the present study, researchers hypothesized that non-elderly adults who were born low birth weight (defined as $<2500$ grams) would be predisposed to developing severe COVID-19 infection relative to those who were born at a normal birth weight. Study authors concluded that low birth weight is an independent risk factor for severe COVID-19 in non-elderly adults. Given the evolving scientific evidence connecting low- and very-low birth weight with adverse health outcomes in adulthood, and the urgent need for risk stratification during the ongoing COVID-19 pandemic, the study aims are intriguing and laudable. However, the authors' results may be unsubstantiated as the authors failed to account for key confounders in their statistical analyses, and there is concern regarding the reliability of birth weight information.

To date, little is known about the effects of low birth weight on COVID-19 complications. Study authors conducted a prospective cohort study in Barcelona where individuals with laboratory-confirmed SARS-CoV-2 infection were followed until admission to the ICU or discharged. Study participants self-reported birth weight and gestational age at delivery. A major concern of this study is the reliability of the birth weight data. Some studies report poor correspondence between officially recorded and self-reported birth weights (Little 1986 Am J Pub Health). Others have found that there is reasonable accuracy (Sanderson et al, $1998 \mathrm{Am} \mathrm{J} \mathrm{Epidemiol).} \mathrm{Importantly,} \mathrm{low}$ birthweight people tend to under-report their birth weight (Kemp et al, 2000 BMJ). In the present study, authors cite only one study in the discussion section to bolster the claim that self-reported birth weight is reliable, however the cited study was conducted among twins in Norway and may not be applicable to singleton adults in Spain. A review article examining reliability of self-reported birth weight found 
"moderate to poor" reliability among elderly adults, particularly without a mother's (or sibling's) recall (Kemp et al, 2000 BMJ).

The complex relationship between low birth weight, gestational age, fetal growth restriction, and cardiopulmonary health in adulthood makes interpretation of the study results difficult. There is evidence, as the authors note, that prematurity and fetal growth restriction are associated with adverse respiratory and cardiovascular outcomes later in life, and that prematurity and fetal growth restriction may cause low birth weight. However, as discussed, it is not clear that self-reported low birth weight is a "reliable and robust surrogate" of prematurity and fetal growth restriction. Low birth weight itself has been strongly linked to adverse health outcomes in adulthood, giving this study question merit. The strongest evidence relates low birth weight to chronic, noncommunicable diseases, particularly cardiovascular and ischemic disease, in men and women. There is, however, evidence that adults with a history of very low birth weight (<1500 grams) and moderately low birth weight (1500-2499 grams) are at increased risk of hospitalization for respiratory illness, including respiratory infections, even after controlling for maternal smoking during pregnancy (Watler et al 2009, Am J Rep Crit Care Med).

In the statistical analyses, authors created a predictive model. Age, sex, smoking status, chronic health conditions, low birth weight, fetal growth restriction, and preterm birth (gestational age at birth $<37$ weeks) were included as possible predictors. In the final predictive model, age, sex, hypertension, and low birth weight were retained. The predictive model was validated in a developmental cohort, which included volunteers in several European countries who self-reported having laboratoryconfirmed SARS-CoV-2 infection. Volunteers were recruited via social media and email and information was obtained via a self-reported survey.

One concern with this final predictive model is that low birth weight alone (not prematurity or fetal growth restriction) were retained in the final model. Low birth weight is commonly confounded by preterm birth, as low birth weight could be a consequence of preterm birth. Furthermore, low socioeconomic status (SES) is a wellknown risk factor for low birth weight and a growing body of literature has shown that low SES is associated with COVID-19 infection, which suggests that these results could be subject to residual confounding by SES. The authors also did not account for smoking history, another significant potential confounder. While the final predictive model did perform well in the validation dataset, there is concern that those volunteers who self-selected in to take the survey are higher SES than the general population, as 
an underlying assumption for this study was that volunteers had access to email and/or the internet. Furthermore, those who self-select into the study may be healthier than the general population.

The authors of the paper are to be applauded for pursuing a plausible hypothesis to better understand risks of severe complications from COVID-19 infection, which to date is only broadly understood. More research into this question is certainly needed. Overall, the reliability of self-reported birth weight and failure to adequately account for SES in the analysis are major concerns of this study and as such, study results should be interpreted with caution. 